Published in final edited form as:

Am J Obstet Gynecol. 2012 October ; 207(4): 326.e1-326.e9. doi:10.1016/j.ajog.2012.06.048.

\title{
Gestational diabetes screening with the new IADPSG guidelines: a cost-effectiveness analysis
}

\author{
John F. MISSION, MD ${ }^{1}$, Mika S. OHNO, MD², Yvonne W. CHENG, MD, MPH ${ }^{3}$, and Aaron B. \\ CAUGHEY, MD, PhD ${ }^{1}$ \\ ${ }^{1}$ Department of Obstetrics \& Gynecology, Oregon Health \& Science University, Portland, OR \\ ${ }^{2}$ Department of Obstetrics \& Gynecology, Santa Clara Valley Medical Center, San Jose, CA \\ ${ }^{3}$ Department of Obstetrics, Gynecology \& Reproductive Sciences, UCSF, San Francisco, CA
}

\begin{abstract}
Objective-This study investigates the cost-effectiveness of gestational diabetes mellitus (GDM) screening using the new IADPSG guidelines.

Study Design-A decision analytic model was built comparing routine screening with the $2 \mathrm{~h}$ OGTT vs. the 1-hour GCT. All probabilities, costs, and benefits were derived from the literature. Base-case, sensitivity analyses, and a Monte Carlo simulation were performed.

Results-Screening with the $2 \mathrm{~h}$ GTT was more expensive, more effective, and cost-effective at $\$ 61,503 / \mathrm{QALY}$. In a one-way sensitivity analysis, the more inclusive IADPSG diagnostic approach remained cost-effective as long an additional $2.0 \%$ or more of patients were diagnosed and treated for GDM.
\end{abstract}

Conclusion-Screening at 24-28 weeks GA under the new IADPSG guidelines with the $2 \mathrm{~h}$ GTT is expensive but cost-effective in improving maternal and neonatal outcomes. How the health care system will provide expanded care to this group of women will need to be examined.

\section{Keywords}

cost-effectiveness; decision analysis; gestational diabetes; 2-hour glucose tolerance test; IADPSG guidelines

Correspondence: John Mission, MD, c/o Oregon Health \& Science University, Department of Obstetrics \& Gynecology, Mail Code L466, 3181 SW Sam Jackson Park Rd. Portland, OR 97239, mission@ ohsu.edu, cell: 650-270-1658.

Presentation Information:

Society for Maternal Fetal Medicine Annual Meeting 2012, Dallas, Texas. Abstract \#257, poster presentation on February 9, 2012.

DISCLOSURE: None of the authors have a conflict of interest

Publisher's Disclaimer: This is a PDF file of an unedited manuscript that has been accepted for publication. As a service to our customers we are providing this early version of the manuscript. The manuscript will undergo copyediting, typesetting, and review of the resulting proof before it is published in its final citable form. Please note that during the production process errors may be discovered which could affect the content, and all legal disclaimers that apply to the journal pertain. 


\section{Introduction}

The prevalence of Gestational Diabetes (GDM) has increased alongside the prevalence of obesity in the United States, with an estimated 6-7\% of pregnant patients carrying a diagnosis of GDM. ${ }^{1}$ GDM is also associated with numerous complications of pregnancy, including higher rates of preeclampsia, operative deliveries, macrosomia, shoulder dystocia, and birth injuries. ${ }^{2}$

Diagnostic criteria for GDM are evolving. The Hyperglycemia and Adverse Pregnancy Outcomes (HAPO) study demonstrated that hyperglycemia at levels below those diagnostic for GDM were associated with adverse maternal and neonatal outcomes. ${ }^{3}$ For this reason, the International Association of Diabetes in Pregnancy Study Groups (IADPSG) convened a workshop conference in 2008 where they recommended using new cutoffs for the 2-hour oral glucose tolerance test (2h OGTT) in GDM screening and diagnosis. The IADPSG selected cutoffs associated with an odds ratio of 1.75 times the mean for outcomes of increased body fat, large-for-gestational-age (LGA), and cord serum C-peptide greater than the 90th centile, yielding the new recommended diagnostic criteria for GDM. ${ }^{4}$ In the U.S., this would mean moving away from a screening test followed by a diagnostic test to a single universal test consisting of a fasting blood glucose followed by the 2-h OGTT using a 75-gm glucose load. Under these new criteria, patients would qualify for a diagnosis of GDM if their 2-h OGTT exceeded one or more of the following thresholds: a fasting glucose above 92, a 1-hour plasma glucose above 180, or a 2-hour plasma glucose above 153.

The adoption of these criteria is controversial. According to these criteria, an estimated $18 \%$ of patients would qualify for a diagnosis of GDM, ${ }^{5}$ potentially adding to the costs of care for many pregnant women in the United States. Prior cost-effectiveness analyses have found universal screening with the 1-hour glucose challenge test (1-h GCT) to be more costeffective than the $2 \mathrm{~h} \mathrm{OGTT}^{6}$ These studies, however, examined the previous WHO criteria used for GDM screening in pregnancy. In addition, two randomized controlled trials published after these studies have demonstrated that treatment of GDM decreases the risk of maternal and neonatal adverse outcomes, ${ }^{7,8}$ with the more recent study demonstrating that treating mild GDM decreased the risks of fetal macrosomia, shoulder dystocia, cesarean delivery, and hypertensive disorders. ${ }^{8}$

The goal of our study was to conduct a cost-effectiveness analysis to determine from a societal standpoint which routine GDM screening method was more cost-effective: universal screening according to current ACOG guidelines with the $1 \mathrm{~h}$ GCT followed by a 3-hour OGTT or the new IADPSG guidelines for the $2 \mathrm{~h}$ OGTT.

\section{Materials and Methods}

A decision-analytic model was designed using TreeAge Pro Software (2011 version, Williamstown, MA) to compare total costs, total maternal and neonatal quality-adjusted life years (QALYs) from a societal perspective for women undergoing routine GDM screening at 24-28 weeks with either the routine $1 \mathrm{~h} \mathrm{GCT}$ or the new IADPSG guidelines for the $2 \mathrm{~h}$ OGTT (Figure 1). Given that current ACOG guidelines recommend universal GDM 
screening, the option of no screening was not investigated and only screening with the $2 \mathrm{~h}$ OGTT and the 1h GCT were compared. As no human subjects were involved in creating this theoretical model, this study was exempt from Institutional Review Board approval. Patients in each strategy were categorized into three groups: patients who would qualify for a GDM diagnosis by the 1-h GCT criteria (Group 1), the additional patients who would be diagnosed with GDM under the new screening guidelines (Group 2), and patients with normal glycemic levels (Group 3).

Maternal outcomes in the model included: preeclampsia, shoulder dystocia, cesarean vs. vaginal delivery, and maternal death. Neonatal outcomes included: macrosomia ( $>4000 \mathrm{~g})$, permanent or transient brachial plexus injury, hypoglycemia, admission to a neonatal intensive care unit (NICU), hyperbilirubinemia, and neonatal death. All probabilities, costs and utilities were derived from the literature. The incremental cost-effectiveness ratio (ICER), the ratio of health care dollars spent to health outcomes obtained, was measured in 2012 \$US per QALY gained. In the US, the range of what is considered cost effective ranges from $\$ 50,000$ to $\$ 100,000$ per QALY. Thus, we considered any ICER below $\$ 50,000$ per QALY as cost effective and any ICER between $\$ 50,000$ and $\$ 100,000$ per QALY as marginally cost effective. Cost-effectiveness thresholds greater than $\$ 100,000$ per QALY were considered not cost effective. ${ }^{9}$

\section{Probabilities}

The baseline probabilities for preeclampsia, cesarean delivery, macrosomia, neonatal death, and NICU admission were derived from the literature. ${ }^{3,10,11}$ (Table 1) For the group of patients who would be diagnosed under the $2 \mathrm{~h}$ OGTT using the new IADPSG guidelines, the baseline probabilities were derived directly from the data for HAPO Group 5, representing the patients within the HAPO study whose glycemic values straddle the new IADPSG guidelines. ${ }^{3,11}$ The baseline probability of falling in Group 1 was obtained from 2008 data on pregnancy hospital discharges in the United States, while the baseline probability for falling in Group 2 was inferred from HAPO study data. ${ }^{1,3}$ As the presence of macrosomia affects the probability of shoulder dystocia, brachial plexus injury, hypoglycemia, and hyperbilirubinemia, these probabilities were derived from a separate body of work that examined these factors independently in patients with GDM. ${ }^{12-14}$ Probabilities for maternal death were also derived from the literature. ${ }^{15}$

To estimate the effect of GDM treatment on these outcomes for patients in Group 1, the baseline probabilities were multiplied by relative risks taken both from a recent metaanalysis on the effects of GDM treatment on pregnancy outcomes as well as the relative risks for treating mild GDM. ${ }^{10,16}$ For patients in Group 2, baseline probabilities were multiplied by the relative risk associated with treatment published in the $2009 \mathrm{RCT}$ on treating mild GDM. ${ }^{10}$

\section{Costs}

All costs were in 2012 US dollars and were inflated using the medical component of the consumer price index. ${ }^{17-24}$ (Table 1) Of note, the cost of treating GDM was set at a baseline of $\$ 1,971$, which included pharmacotherapy, antenatal visits, ancillary diabetes-related 
visits, and antepartum fetal surveillance. ${ }^{17}$ The cost of GDM diagnosis was accounted for and included the direct cost of test materials and analysis as well as indirect costs of travel and opportunity costs for the patient. ${ }^{18}$ The costs accounted for neither antepartum admissions for GDM-related conditions nor broader indirect costs. ${ }^{17}$ Because maternal mortality is difficult to quantify with a point estimate as the values vary based on the circumstances of death, a baseline of $\$ 100,000$ was used and was subject to sensitivity analysis. ${ }^{25}$ For brachial plexus injury, adjusted published costs were $\$ 17,322$ for permanent injury and $\$ 1939$ for transient injury. ${ }^{24}$ All costs were subject to sensitivity analysis.

\section{Utilities}

All utilities were derived from the literature and include utilities from the maternal and neonatal perspective. (Table 1) The utility of a vaginal delivery was assumed to be 1 and the utility of a cesarean delivery was set to a baseline of 0.99 based on previously published research on women's preferences in mode of delivery. ${ }^{26-28}$ Maternal death by definition was set to a utility of 0 . The utility of a neonatal death from the maternal perspective was set at a baseline of 0.92 , the published maternal utility of a miscarriage, and was applied over the maternal lifetime. ${ }^{27,29}$ The utility of a neonatal death from the neonatal perspective was by definition 0 and applied over the neonatal lifetime. The neonatal utility of a transient brachial plexus injury was set to a baseline of 0.99 based on "brachial plexus injury that resolves within 2 months." ${ }^{24}$ For a permanent brachial plexus injury, a conservative value of 0.6 was used based on a published value for mild to moderate injury, compared with a utility of 0.45 for a severe injury. ${ }^{24}$ These utilities were applied over the neonatal lifetime. As there are no published utilities on short-term neonatal consequences such as hypoglycemia, NICU admission, or hyperbilirubinemia, these utilities were set to a conservative baseline value of $1 .{ }^{27}$ Utilities were calculated over the course of maternal life expectancy (56.1 years) and neonatal life expectancy $(77.2 \mathrm{y})^{30}$ at a discount rate of $3 \% .^{9}$ All utilities were subject to sensitivity analysis.

\section{Analysis}

Baseline analysis compared routine GDM screening with the $1 \mathrm{~h} \mathrm{GCT}$ vs. the $2 \mathrm{~h}$ OGTT to estimate differences in maternal and neonatal outcomes, total costs, and QALYs for each strategy as well as the incremental cost-effectiveness ratio (ICER).

Sensitivity analysis is a statistical tool that helps clarify key variables that most influence outcomes in the model and test the robustness of the model. A threshold value marks the point at which change in a variable would alter the conclusion. We tested the robustness of our model by performing univariate sensitivity analysis on all inputs and also introduced an additional variable in the sensitivity analysis to represent the percent efficacy of treatment, attempting to account for practice variations that can result in a range of outcomes with treatment.

Monte Carlo Analysis is a tool that can incorporate uncertainty into the baseline stochastic model by sampling the underlying distributions for each input. One simulation represents a woman undergoing routine GDM screening with either the $1 \mathrm{~h} \mathrm{GCT}$ or the $2 \mathrm{~h}$ OGTT. Her probabilities and costs are randomly chosen from a pre-specified distribution determined 
from the literature, and this simulation is repeated with a different set of randomly chosen values, with the aggregate representing a theoretical cohort of random women. ${ }^{27}$ Based on these simulations, an acceptability curve can be constructed showing the probability of achieving cost-effectiveness. To adopt the deterministic model to a stochastic one for the Monte Carlo simulation, costs were modeled using a gamma distribution, and probabilities were modeled using a beta distribution.

\section{Results}

Maternal and neonatal outcomes associated with GDM screening for a theoretical cohort of 100,000 women were estimated (Table 2). Screening with the $2 \mathrm{~h}$ OGTT decreased all maternal outcomes, including preeclampsia, cesarean delivery, and shoulder dystocia. In addition, using the $2 \mathrm{~h}$ OGTT decreased the neonatal outcomes of macrosomia, hypoglycemia, hyperbilirubinemia, and transient brachial plexus injury. Using the $2 \mathrm{~h}$ OGTT did not have an effect on permanent brachial plexus injury.

The baseline cost-effectiveness analysis results show that screening with the $2 \mathrm{~h}$ OGTT is more expensive at $\$ 12,201$ vs. $\$ 12,078$ with $1 \mathrm{~h}$ GCT screening but more effective shown by higher QALYs at 56.713799 vs 56.711790 with the $1 \mathrm{~h} \mathrm{GCT} \mathrm{(Table} \mathrm{3).} \mathrm{The} \mathrm{incremental} \mathrm{cost/}$ QALY is cost-effective (below the cost-effectiveness threshold of $\$ 100,000 / \mathrm{QALY}$ ) at \$61,503/QALY.

\section{Sensitivity analysis}

Univariate sensitivity analyses were conducted on all probabilities, costs, and utilities. The model remained robust across reasonable ranges for all of the variables with few exceptions. Examining the additional percentage of pregnant women who would be diagnosed with GDM under the IADPSG guidelines (Group 2) revealed that screening with the $2 \mathrm{~h}$ OGTT was cost-effective (below $\$ 100,000 / \mathrm{QALY}$ ) as long as an additional $2.04 \%$ of screened patients would be newly diagnosed with GDM under the new guidelines (Figure 2a).

Examining costs, the only costs that made GDM screening with the $2 \mathrm{~h}$ OGTT no longer cost-effective were the incremental cost of GDM treatment and the cost of the 2h OGTT. (Figure $2 \mathrm{~b}$ and 2c). Screening with the $2 \mathrm{~h}$ OGTT was cost-effective (below $\$ 100,000 /$ QALY) as long as the cost to treat GDM was less than \$2630 (Figure 2b) and as long as the cost of the $2 \mathrm{~h}$ OGTT was less than $\$ 175.74$ (Figure 2c). To examine the interaction of the costs of the 1-hour and 2-hour screening tests, a two-way sensitivity analyses comparing these two variables were performed and showed that the model remained cost-effective as long as the incremental cost of 2-hour screening over 1-hour screening was less than $\$ 92.56$. (Figure 3a)

Univariate sensitivity analysis on the efficacy of treatment showed that at the baseline cost to treat GDM of $\$ 1971$, screening with the 2h OGTT was cost-effective as long as GDM treatment was at least $74.9 \%$ efficacious. (Figure 2d) Since the efficacy of treatment also affected the cost-effectiveness threshold (the lower the efficacy, the lower the cost-threshold were it was no longer cost-effective to screen with the $2 \mathrm{~h}$ OGTT), two-way sensitivity analysis was performed on treatment efficacy vs. incremental cost to treat GDM. (Figure 3b) 
At $100 \%$ treatment efficacy, the cost to treat could be as high as $\$ 2,630$ and still be costeffective; this represents the baseline cost-effectiveness threshold. As the treatment efficacy decreased, the upper limit on the cost to treat GDM that was still cost effective decreased linearly.

Sensitivity analysis was performed to further examine the outcomes that had the greatest impact on the cost-effectiveness of screening with the $2 \mathrm{~h}$ OGTT. The reduction in two outcomes showed an effect on the cost-effectiveness of screening with the 2h OGTT: the reduction in rates of preeclampsia and cesarean section in Group 2 from GDM diagnosis and from subsequent treatment under the new $2 \mathrm{~h}$ OGTT compared to no diagnosis or treatment with the 1h OGTT. Two-way sensitivity analysis was performed on the probabilities of preeclampsia in Group 2 with GDM diagnosis and treatment under the 2h OGTT vs. $1 \mathrm{~h}$ GCT screening without GDM diagnosis or treatment. The model remained cost-effective as long as the incremental reduction in the preeclampsia rate was greater than $0.55 \%$ with 2hour OGTT GDM diagnosis and subsequent treatment (Figure 3c). Likewise, using two-way sensitivity analysis to compare the rates of cesarean with and without treatment in Group 2 with and without treatment under the $2 \mathrm{~h}$ OGTT vs. the $1 \mathrm{~h}$ GCT, the model was costeffective as long as the incremental reduction in the cesarean section rate was greater than $2.7 \%$ with $2 \mathrm{~h}$ OGTT screening, GDM diagnosis and subsequent GDM treatment in this group (Figure 3d).

A Monte Carlo simulation analysis was performed to simulate the outcome of 10,000 random pregnant women undergoing GDM screening. Based on these simulations, a willingness-to-pay acceptability curve showed that a cost-effectiveness threshold of $\$ 100,000 /$ QALY yielded a $75.7 \%$ probability that screening with the $2 \mathrm{~h}$ OGTT would be cost-effective (Figure 4).

\section{Comment}

Our decision analytic model showed that screening with the $2 \mathrm{~h}$ GTT using the new IADPSG guidelines was cost-effective as long as the cost to treat GDM was less than $\$ 2,630$ or when the efficacy of treatment met at least $74.9 \%$ of its expected estimate. In addition, the cost effectiveness of whether to diagnose GDM with the 2h OGTT and the new IADPSG guidelines was dependent on the effect of treatment in reducing preeclampsia and cesarean delivery. Finally, the model remained cost-effective if at least an additional $2.0 \%$ of patients were diagnosed with GDM under the new IADPSG screening guidelines, a threshold that is far lower than the expected percentage of patients who would be diagnosed with GDM. ${ }^{5}$

Prior cost analyses have investigated the effectiveness of using the 2-hour OGTT in comparison to the 1-hour GCT using the new IADPSG guidelines. ${ }^{18,31}$ One prior analysis looking at using the 2-hour OGTT compared the cost of the 2-hour OGTT regime to the costs of the 1-hour GCT screening but include neither utilities for perinatal outcomes associated with GDM nor costs for these outcomes. ${ }^{18}$ Another cost analysis found that screening with the 2-hour OGTT was not cost-effective when limiting their analysis to pregnancy. ${ }^{31}$ These results conflict with the findings of our study to some degree. Our analysis looked specifically at patients in Group 5 of the HAPO Study, whose 2h OGTT 
results more accurately reflect the additional patients diagnosed with GDM under the new IADPSG guidelines. ${ }^{3}$ Our analysis accounted for additional neonatal outcomes that are associated with GDM, including macrosomia, neonatal hyperbilirubinemia, and hypoglycemia. We also accounted for the effects of macrosomia on these outcomes. ${ }^{12}$

The aforementioned cost analysis also differed in the relative risk reduction values used for the outcomes of cesarean section and preeclampsia. Their analyses assumed that the benefits of GDM treatment would translate to the additional patients who would newly qualify for a GDM diagnosis under the new IADPSG criteria. ${ }^{31}$ To calculate the effects of treatment on cesarean section and other outcomes, this group used the results from a recent meta-analysis on perinatal outcomes after GDM treatment to estimate the effects of treatment on rates of cesarean section, which found no difference in the rates of cesarean section in patients with GDM. ${ }^{16}$ Our analysis extrapolated the effect of treatment on cesarean section by using the results of the RCT examining treatment of mild gestational diabetes, which found a relative risk reduction of 0.79 in cesarean section rate with GDM treatment, ${ }^{10}$ and applying these results to patients in Group 2 of our analysis. As indicated in our sensitivity analyses, an incremental reduction in the cesarean section rate by $2.7 \%$ in Group 2 is required for our model to be cost-effective, and if there were no difference in cesarean rates with GDM treatment, screening with the $2 \mathrm{~h}$ OGTT would not be cost effective under our model.

Examining preeclampsia reduction with treatment, the prior study also derived a value 0.65 for the relative risk of preeclampsia with GDM treatment using data from a Cochrane review on preeclampsia rates after GDM treatment. ${ }^{32}$ This value is more conservative than the RR of 0.46 , which was also derived from the study investigating treating mild gestational diabetes and applied to group 2 of our analysis. ${ }^{8}$ According to our sensitivity analysis on preeclampsia rates, however, only an absolute risk reduction in preeclampsia rates by $0.55 \%$ or .0055 is required to maintain cost-effectiveness in our model. With a baseline preeclampsia risk of.0679, this would correspond to an RR of 0.92 required for our model to still be cost effective. Thus, using the more conservative RR from their analysis would still result in the $2 \mathrm{~h}$ OGTT screening to be cost effective over the $1 \mathrm{~h}$ GTT.

Interestingly, when broadening their analyses to include an intervention to decrease the likelihood of progression to Type 2 Diabetes Mellitus (T2DM), screening with the 2-hour OGTT became cost effective using their model. ${ }^{31}$ While one RCT has shown benefit from an intervention to delay the onset of T2DM,${ }^{33}$ rates of postpartum glucose follow-up in patients with GDM is poor, ${ }^{34}$ even despite an intervention involving intensive patient counseling to improve postpartum glucose follow-up in patients with GDM. ${ }^{35}$ Our model did not account for such a benefit in reducing the progression of GDM to T2DM, but if this finding holds true, then the benefit gained from preventing future diagnoses of T2DM could potentially further increase the cost-effectiveness of our model.

Several limitations limit the interpretations of our findings. First, decision analysis has its inherent limitations in simulating real-world outcomes. In our model, some neonatal outcomes are modeled as discrete outcomes that cannot coexist. For example, a neonate in our model cannot have both hypoglycemia and hyperbilirubinemia even though both outcomes can occur in real world situations. Adding permutations for all possible 
combinations of outcomes studied would make decision analytic models too unwieldy for practical use. Our study did, however, account for the association of variables that are very closely related (i.e. macrosomia, shoulder dystocia, and brachial plexus injury). In addition, we were unable to model the potential effects of maternal GDM on long-term outcomes such as future obesity and T2DM risk as previously discussed. If such findings were incorporated into our model, the findings would be even more robust.

The findings of our study also depend on the effectiveness of GDM treatment in reducing rates of maternal and neonatal complications. The cost effectiveness of our model was particularly sensitive to reducing cesarean section rates and preeclampsia rates in patients who would be incrementally diagnosed with GDM under the $2 \mathrm{~h}$ OGTT as previously described in our sensitivity analysis. Although we inferred the benefits of treatment from a randomized controlled trial investigating the benefits of GDM treatment on patients with mild gestational diabetes so as to more accurately reflect a population with less severe hyperglycemia, ${ }^{10}$ no current data exists that examines the benefits of GDM treatment on maternal and neonatal outcomes for patients who would be incrementally diagnosed with GDM under the new IADPSG guidelines.

Another limitation involves our inability to model certain costs in our analysis. Antenatal admissions can add to the overall cost of managing women with GDM but were not included in this analysis as the effects of GDM treatment on antenatal hospitalization rates are unclear. One cost-consequence analysis of treating patients with "glucose intolerance of pregnancy" by pre- 1998 World Health Organization (WHO) criteria found no difference in antenatal inpatient admission rates. ${ }^{36}$ Given that glycemic levels in patients may be less severe in patients who would be incrementally diagnosed under the new screening criteria, it is unclear if rates of antenatal admission would be higher with GDM treatment.

Furthermore, while the costs of screening accounted for the opportunity cost of lost time from work, we were unable to model the costs of increased antenatal surveillance for patients. Finally, the values used for costs of GDM treatment in our study come from a review published in 2000 as more recent data on the costs of GDM treatment in the US were lacking in the literature. As our model was sensitive to the costs of GDM treatment, more recent data would be useful in assuring the cost-effectiveness of screening with the IADPSG guidelines.

Although our study found that universal screening with the newly proposed IADPSG guidelines for the 2-hour OGTT is more costly, our study contributes to a body of literature that suggests that this GDM screening method may be cost-effective under certain circumstances. Our model is robust over a wide range of inputs for the parameters we investigated with several notable exceptions that warrant further study. As the cost of treatment is one of the inputs to which our model is sensitive, research into methods to decrease costs in management, supplies, and treatments would be an important step in increasing the cost-effectiveness of GDM screening. The cost effectiveness of our model is also dependent on a reduction in both preeclampsia and cesarean section rates resultant from a new GDM diagnosis with the $2 \mathrm{~h}$ OGTT and subsequent treatment. Further studies to demonstrate benefit the benefit of treatment in patients who would be diagnosed with newly suggested GDM criteria are needed. Furthermore, the emerging field of developmental 
origins of human disease suggests that glucose intolerance during pregnancy may be associated with long-term outcomes such as T2DM, hypertension, and obesity. Long-term studies to investigate the potential link between GDM and adult outcomes for the children of patients with GDM are needed. Finally, our model demonstrates that only an additional 2\% of patients need to be incrementally diagnosed with GDM to still remain cost-effective. This is much lower than the anticipated total $18 \%$ of patients who would be diagnosed with GDM under the new guidelines, ${ }^{5}$ and as the incidence of obesity swells in the US, the additional percentage of patients diagnosed under the IADPSG guidelines will continue to increase.

\section{Acknowledgments}

Dr. Yvonne Cheng is supported by the UCSF Women's Reproductive Health Research Career Development Award, NIH, the Eunice Kennedy Shriver National Institute of Child Health and Human Development (K12 HD001262)

\section{References}

1. Wier, LM.; Witt, E.; Burgess, J.; Elixhauser, A. Healthcare Cost and Utilization Project (HCUP) Statistical Briefs. Rockville MD: 2006. Hospitalizations Related to Diabetes in Pregnancy, 2008: Statistical Brief \#102.

2. Hillier TA, Vesco KK, Pedula KL, Beil TL, Whitlock EP, Pettitt DJ. Screening for gestational diabetes mellitus: a systematic review for the U.S. Preventive Services Task Force. Ann Intern Med. 2008; 148:766-75. [PubMed: 18490689]

3. Metzger BE, Lowe LP, Dyer AR, et al. Hyperglycemia and adverse pregnancy outcomes. N Engl J Med. 2008; 358:1991-2002. [PubMed: 18463375]

4. Metzger BE, Gabbe SG, Persson B, et al. International association of diabetes and pregnancy study groups recommendations on the diagnosis and classification of hyperglycemia in pregnancy. Diabetes Care. 2010; 33:676-82. [PubMed: 20190296]

5. Landon MB, Gabbe SG. Gestational diabetes mellitus. Obstet Gynecol. 2011; 118:1379-93. [PubMed: 22105269]

6. Poncet B, Touzet S, Rocher L, Berland M, Orgiazzi J, Colin C. Cost-effectiveness analysis of gestational diabetes mellitus screening in France. Eur J Obstet Gynecol Reprod Biol. 2002; 103:122-9. [PubMed: 12069733]

7. Crowther CA, Hiller JE, Moss JR, McPhee AJ, Jeffries WS, Robinson JS. Effect of treatment of gestational diabetes mellitus on pregnancy outcomes. N Engl J Med. 2005; 352:2477-86. [PubMed: 15951574]

8. Landon MB. Is there a benefit to the treatment of mild gestational diabetes mellitus? Am J Obstet Gynecol. 2010; 202:649-53. [PubMed: 20510966]

9. Caughey AB. Cost-effectiveness analysis of prenatal diagnosis: methodological issues and concerns. Gynecol Obstet Invest. 2005; 60:11-8. [PubMed: 15692215]

10. Landon MB, Spong CY, Thom E, et al. A multicenter, randomized trial of treatment for mild gestational diabetes. N Engl J Med. 2009; 361:1339-48. [PubMed: 19797280]

11. Yogev, Chen, Hod, et al. Hyperglycemia and Adverse Pregnancy Outcome (HAPO) study: preeclampsia. Am J Obstet Gynecol. 2010; 202:255 e1-7. [PubMed: 20207245]

12. Esakoff TF, Cheng YW, Sparks TN, Caughey AB. The association between birthweight $4000 \mathrm{~g}$ or greater and perinatal outcomes in patients with and without gestational diabetes mellitus. Am J Obstet Gynecol. 2009; 200:672 e1-4. [PubMed: 19376489]

13. Rouse DJ, Owen J. Prophylactic cesarean delivery for fetal macrosomia diagnosed by means of ultrasonography--A Faustian bargain? Am J Obstet Gynecol. 1999; 181:332-8. [PubMed: 10454678]

14. Ohno MS, Sparks TN, Cheng YW, Caughey AB. Treating mild gestational diabetes mellitus: a cost-effectiveness analysis. Am J Obstet Gynecol. 2011; 205:282 e1-7. [PubMed: 22071065] 
15. Clark SL, Belfort MA, Dildy GA, Herbst MA, Meyers JA, Hankins GD. Maternal death in the 21st century: causes, prevention, and relationship to cesarean delivery. Am J Obstet Gynecol. 2008; 199:36 e1-5. discussion 91-2 e7-11. [PubMed: 18455140]

16. Horvath K, Koch K, Jeitler K, et al. Effects of treatment in women with gestational diabetes mellitus: systematic review and meta-analysis. BMJ. 2010; 340:c1395. [PubMed: 20360215]

17. Kitzmiller JL. Cost analysis of diagnosis and treatment of gestational diabetes mellitus. Clin Obstet Gynecol. 2000; 43:140-53. [PubMed: 10694996]

18. Meltzer SJ, Snyder J, Penrod JR, Nudi M, Morin L. Gestational diabetes mellitus screening and diagnosis: a prospective randomised controlled trial comparing costs of one-step and two-step methods. BJOG. 2010; 117:407-15. [PubMed: 20105163]

19. Meads CA, Cnossen JS, Meher S, et al. Methods of prediction and prevention of pre-eclampsia: systematic reviews of accuracy and effectiveness literature with economic modelling. Health Technol Assess. 2008; 12:iii-iv. 1-270.

20. Bost BW. Cesarean delivery on demand: what will it cost? Am J Obstet Gynecol. 2003; 188:141821. discussion 21-3. [PubMed: 12824972]

21. Lloyd A, Townsend C, Munro V, Twena N, Nielsen S, Holman A. Cost-effectiveness of insulin aspart compared to human insulin in pregnant women with type 1 diabetes in the UK. Curr Med Res Opin. 2009; 25:599-605. [PubMed: 19232034]

22. Suresh GK, Clark RE. Cost-effectiveness of strategies that are intended to prevent kernicterus in newborn infants. Pediatrics. 2004; 114:917-24. [PubMed: 15466085]

23. Phibbs CS, Schmitt SK. Estimates of the cost and length of stay changes that can be attributed to one-week increases in gestational age for premature infants. Early Hum Dev. 2006; 82:85-95. [PubMed: 16459031]

24. Culligan PJ, Myers JA, Goldberg RP, Blackwell L, Gohmann SF, Abell TD. Elective cesarean section to prevent anal incontinence and brachial plexus injuries associated with macrosomia--a decision analysis. Int Urogynecol J Pelvic Floor Dysfunct. 2005; 16:19-28. discussion. [PubMed: 15647962]

25. Grobman WA, Peaceman AM, Socol ML. Cost-effectiveness of elective cesarean delivery after one prior low transverse cesarean. Obstet Gynecol. 2000; 95:745-51. [PubMed: 10775741]

26. Angeja AC, Washington AE, Vargas JE, Gomez R, Rojas I, Caughey AB. Chilean women's preferences regarding mode of delivery: which do they prefer and why? BJOG. 2006; 113:1253-8. [PubMed: 17014679]

27. Kaimal AJ, Little SE, Odibo AO, et al. Cost-effectiveness of elective induction of labor at 41 weeks in nulliparous women. Am J Obstet Gynecol. 2011; 204:137 e1-9. [PubMed: 20965482]

28. Caughey AB, Angeja AC, Vargas JE, Gomez R, Washington AE. Quantitative evaluation of preference for mode of delivery in pregnant Chilean women. Medical decision making : an international journal of the Society for Medical Decision Making. 2003; 23

29. Kuppermann M, Nease RF, Learman LA, Gates E, Blumberg B, Washington AE. Procedurerelated miscarriages and Down syndrome-affected births: implications for prenatal testing based on women's preferences. Obstet Gynecol. 2000; 96:511-6. [PubMed: 11004350]

30. Heron, M.; Hoyert, DL.; Murphy, SL.; Xu, J.; Kochanek, KD.; Tejada-Vera, B. National vital statistics reports. Vol. 57. from the Centers for Disease Control and Prevention, National Center for Health Statistics, National Vital Statistics System; 2009. Deaths: final data for 2006; p. 1-134.

31. Werner EF, Pettker CM, Zuckerwise L, et al. Screening for gestational diabetes mellitus: are the criteria proposed by the international association of the diabetes and pregnancy study groups costeffective? Diabetes Care. 2012; 35:529-35. [PubMed: 22266735]

32. Alwan N, Tuffnell DJ, West J. Treatments for gestational diabetes. Cochrane database of systematic reviews (Online). 2009 CD003395.

33. Ratner RE, Christophi CA, Metzger BE, et al. Prevention of diabetes in women with a history of gestational diabetes: effects of metformin and lifestyle interventions. The Journal of clinical endocrinology and metabolism. 2008; 93:4774-9. [PubMed: 18826999]

34. Stasenko M, Cheng YW, McLean T, Jelin AC, Rand L, Caughey AB. Postpartum follow-up for women with gestational diabetes mellitus. Am J Perinatol. 2010; 27:737-42. [PubMed: 20440668] 
35. Stasenko M, Liddell J, Cheng YW, Sparks TN, Killion M, Caughey AB. Patient counseling increases postpartum follow-up in women with gestational diabetes mellitus. Am J Obstet Gynecol. 2011; 204:522 e1-6. [PubMed: 21514559]

36. Moss JR, Crowther CA, Hiller JE, Willson KJ, Robinson JS. Costs and consequences of treatment for mild gestational diabetes mellitus - evaluation from the ACHOIS randomised trial. BMC Pregnancy Childbirth. 2007; 7:27. [PubMed: 17963528] 


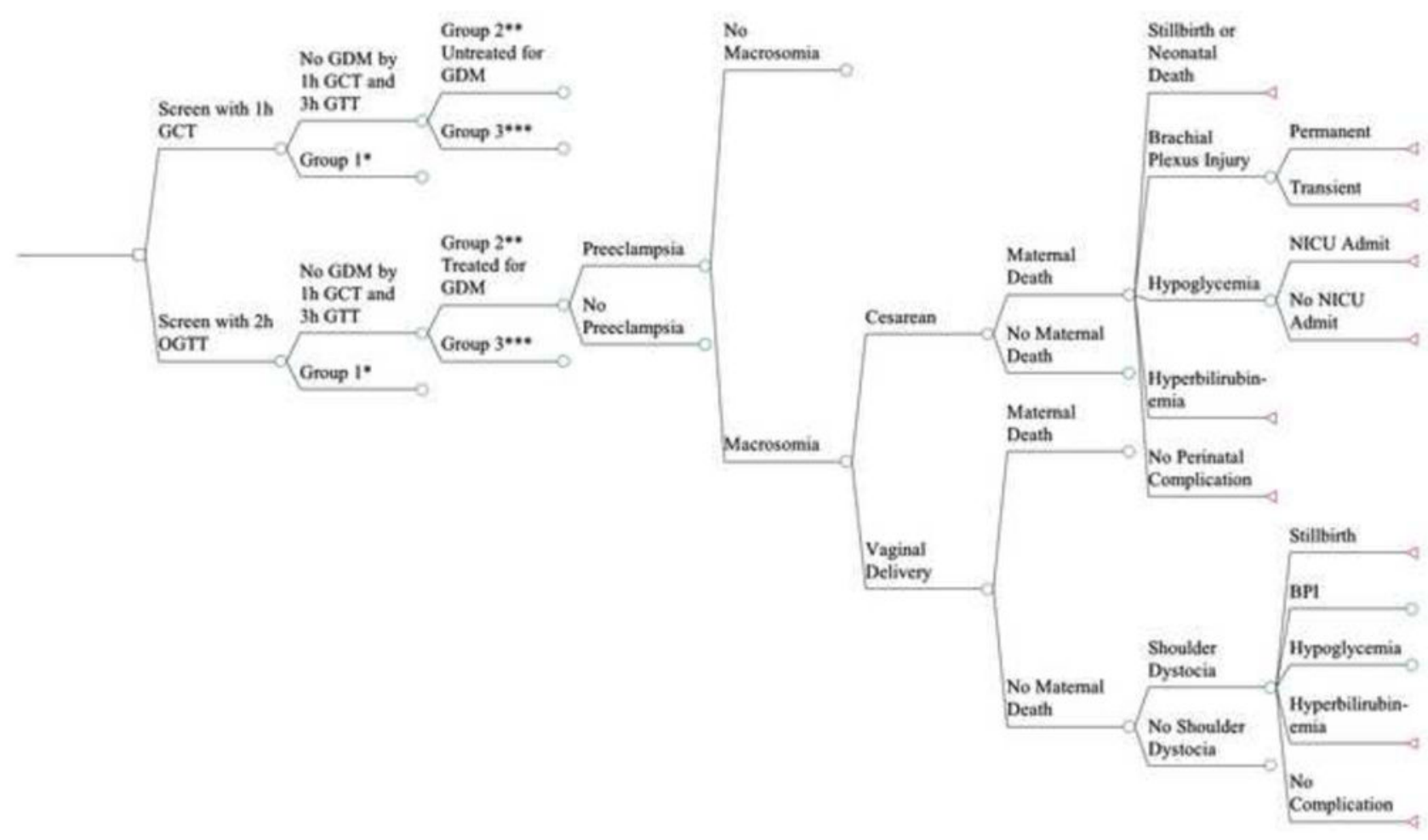

Figure 1.

The decision analytic model comparing universal screening with the $2 \mathrm{~h}$ OGTT vs. the $1 \mathrm{~h}$ GCT. Not all branches are shown to facilitate display. Lines that do not terminate in a triangle indicate they are collapsed to facilitate display and are the same as branches that are already open. 
a

1-Way Sensitivity Analysis:

Percentage of Additional GDM

Diagnoses with 2h OGTT

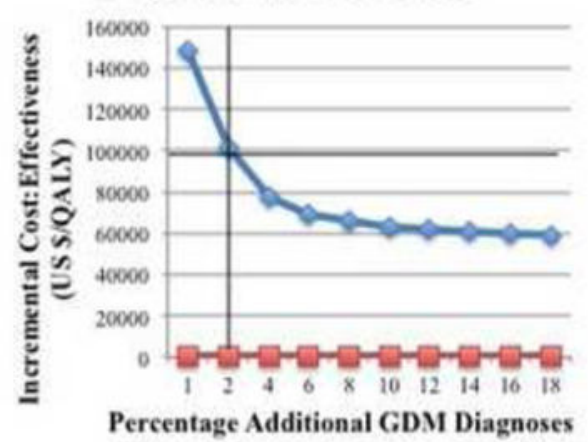

c

\section{1-Way Sensitivity Analysis:}

Cost to Treat GDM

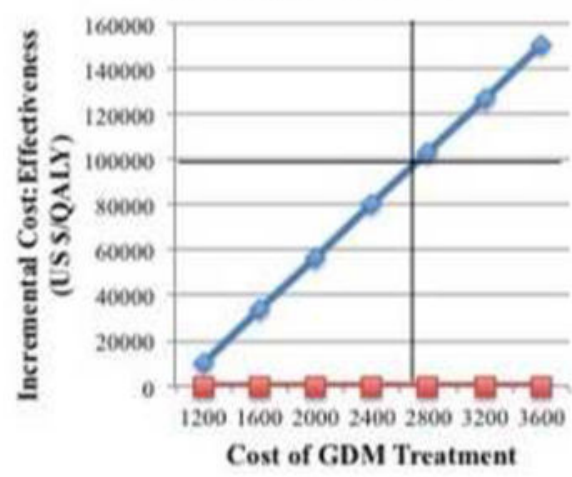

b

\section{1-Way Sensitivity Analysis:} Cost of 2h OGTT

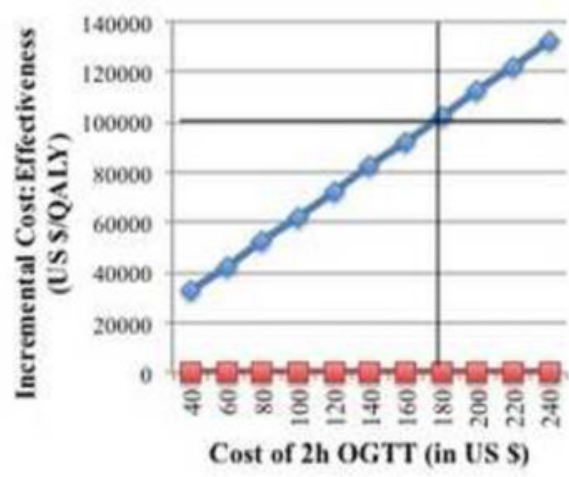

d

1-Way Sensitivity Analysis: Efficacy of Treatment

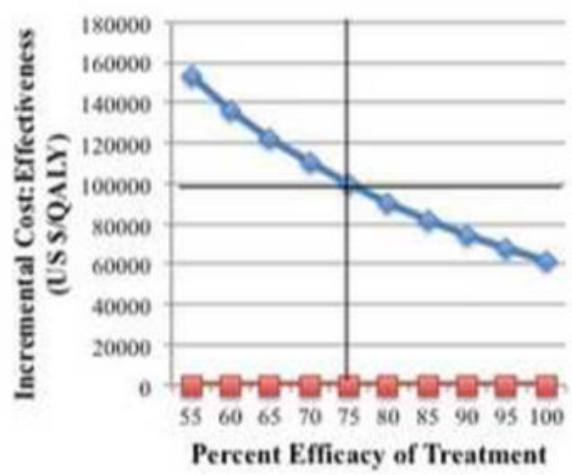

Screen with 1h GCT

Screen with $2 \mathrm{~h}$ GTT

Figure 2.

Univariate Sensitivity Analyses

a. One-way sensitivity analysis of the percentage of additional GDM diagnoses with the $2 \mathrm{~h}$ OGTT. Screening with the $2 \mathrm{~h}$ OGTT was cost-effective (below the cost-effectiveness threshold of $\$ 100,000 / \mathrm{QALY}$ ) as long as an additional $2.04 \%$ of patients would be diagnosed with GDM under the new IADPSG guidelines.

b. One-way sensitivity analysis of the cost of $2 \mathrm{~h}$ OGTT. Screening with the $2 \mathrm{~h}$ OGTT was cost-effective as long as the cost of the $2 \mathrm{~h}$ OGTT was less than $\$ 175.74$.

c. One-way sensitivity analysis of the cost of GDM treatment. Screening with the $2 \mathrm{~h}$ OGTT was cost-effective as long as the cost of GDM treatment was less than $\$ 1971$. 
d. One-way sensitivity analysis of the efficacy of treatment. Screening with the $2 \mathrm{~h}$ OGTT was cost-effective as long as treatment met more than $74.9 \%$ of its reported treatment efficacy. 
a

\section{2-Way Sensitivity Analysis: Costs of $1 \mathrm{~h}$ GCT vs $2 \mathrm{~h}$ OGTT}

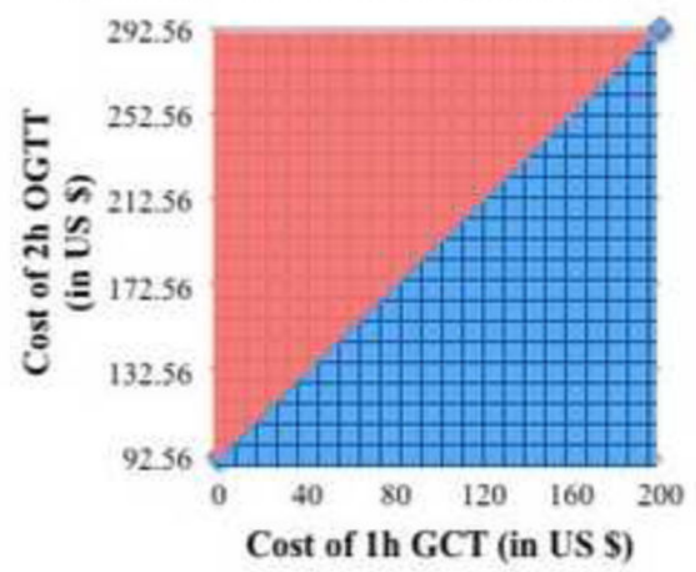

$\mathbf{c}$

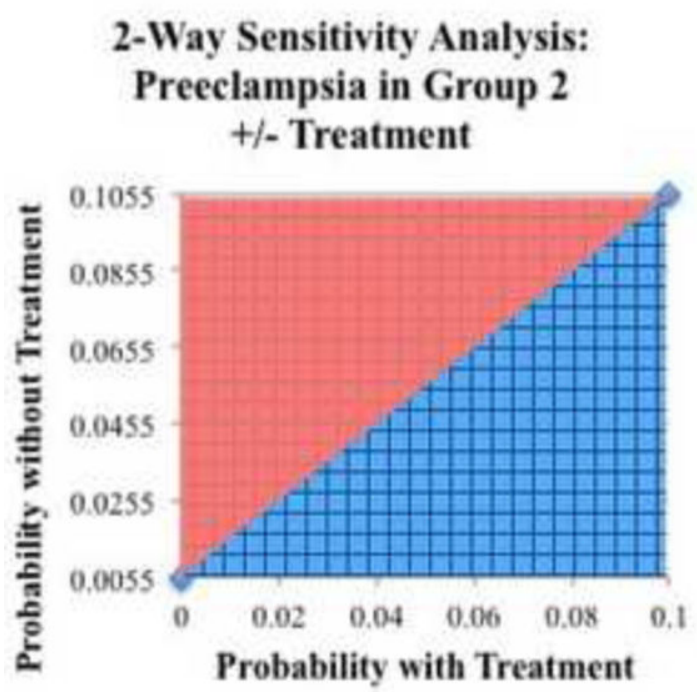

b

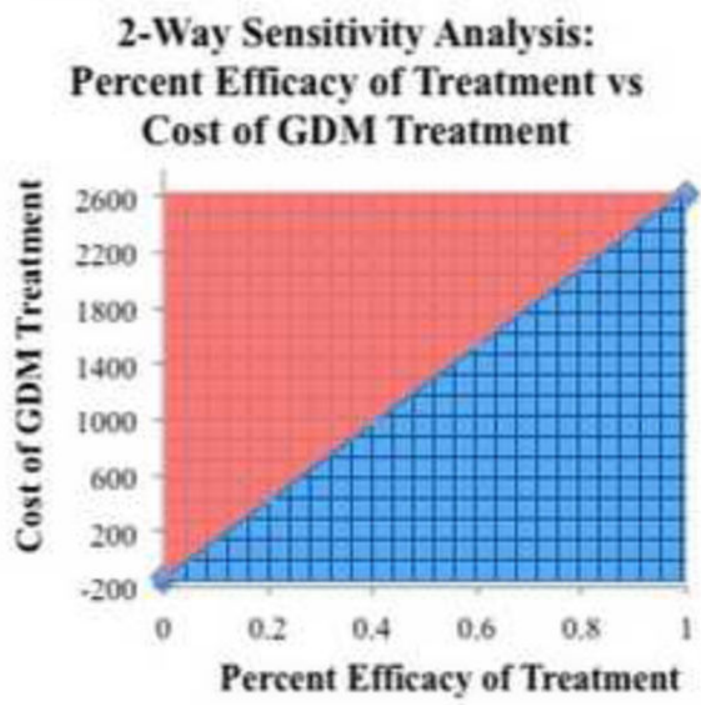

d

2-Way Sensitivity Analysis: Cesarean Section in Group 2 + - Treatment

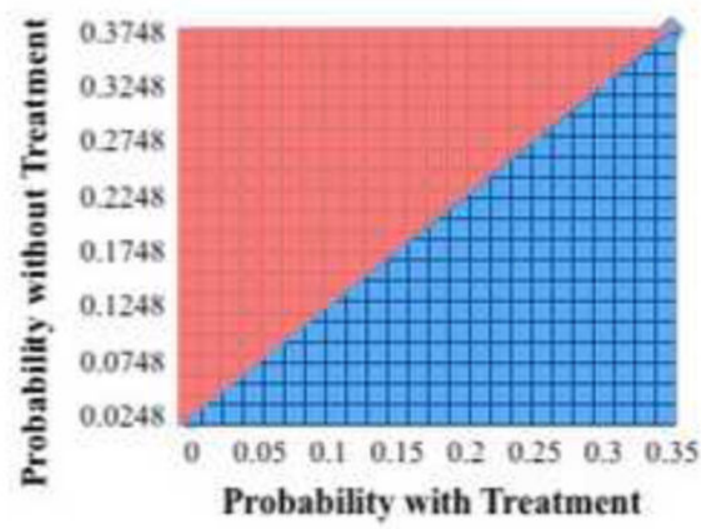

Figure 3.

Two-Way Sensitivity Analyses

a. Two-way senstitivity analysis on the cost of the $1 \mathrm{~h} \mathrm{GCT}$ vs. the cost of the $2 \mathrm{~h}$ OGTT. The blue-hatch region represents where screening with the 2-h OGTT is cost effective (below the cost-effectiveness threshold of $\$ 100,000 / \mathrm{QALY})$. The model remained cost-effective at an incremental cost for the $2 \mathrm{~h}$ OGTT over the $1 \mathrm{~h}$ GTT of less than $\$ 92.56$

b. Two-way senstitivity analysis on the percent efficacy of treatment vs. the cost of GDM treatment. The blue-hatch region represents where screening with the $2 \mathrm{~h}$ OGTT is cost effective. At the baseline GDM treatment cost of $\$ 1971$, 2h OGTT screening was cost 
effective with treatment efficacy as low as $74.9 \%$. At $100 \%$ efficacy, the cost of GDM treatment can be as high as $\$ 2630$ and still be cost-effective.

c. Two-way senstitivity analysis on the probability of preeclampsia in Group 2 with vs. without treatment. Screening with the $2 \mathrm{~h}$ OGTT was cost-effecive as long as the incremental reduction in preeclamsia was greater than $0.55 \%$ in Group 2 as a result of GDM diagnosis and treatment

d. Two-way senstitivity analysis on the probability of cesarean section in Group 2 with vs. without treatment. Screening with the $2 \mathrm{~h}$ OGTT was cost-effective as long as the incremental reduction in cesarean section was greater than $2.7 \%$ as a result of GDM diagnosis and treatment. 


\section{Monte Carlo Analysis: Acceptability Curve}

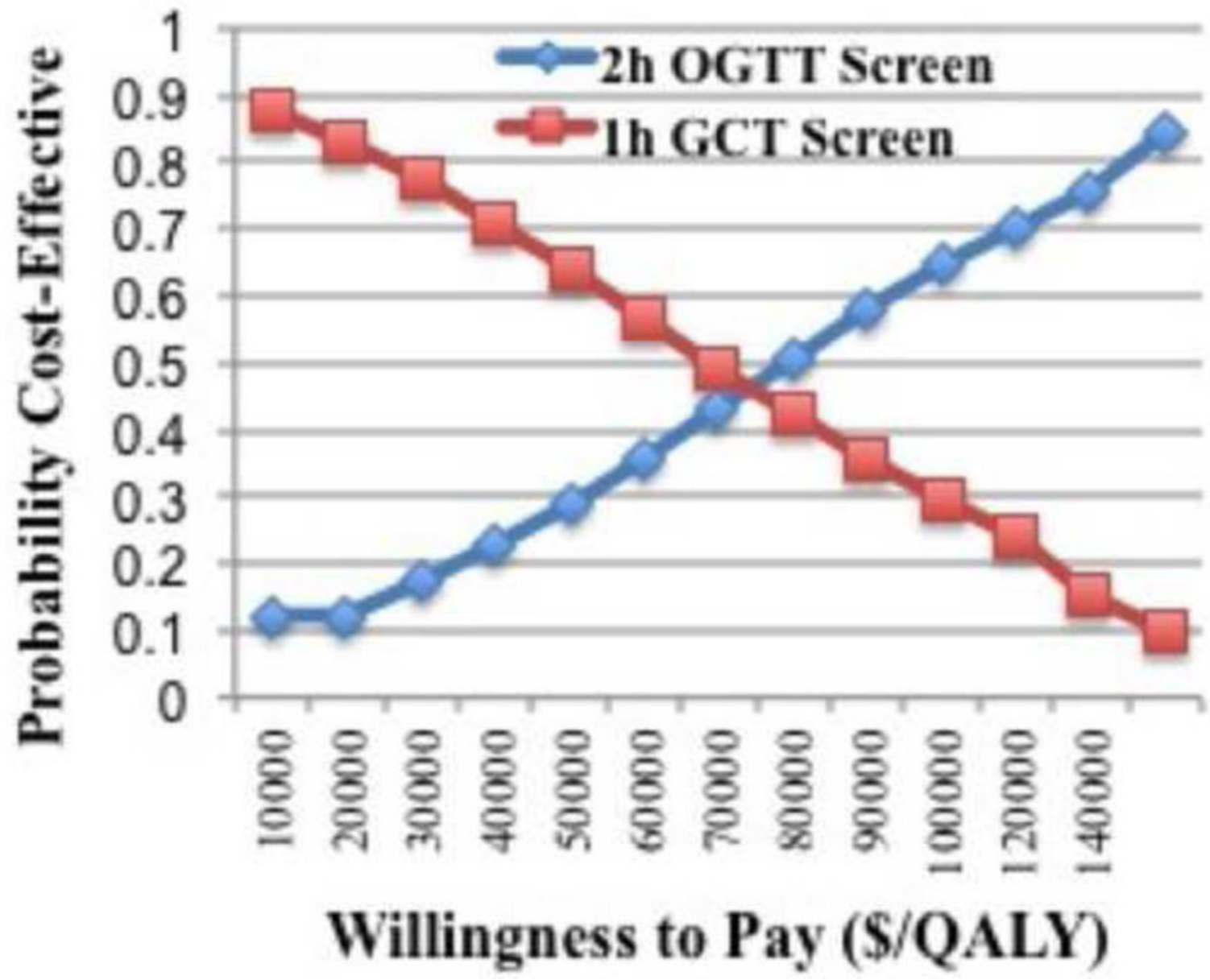

Figure 4.

Monte Carlo simulation of 10,000 random women showing there is a $75.7 \%$ probability that treatment is cost-effective at a threshold of $\$ 100,000 /$ quality-adjusted life year. 


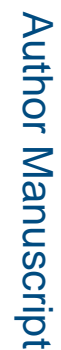

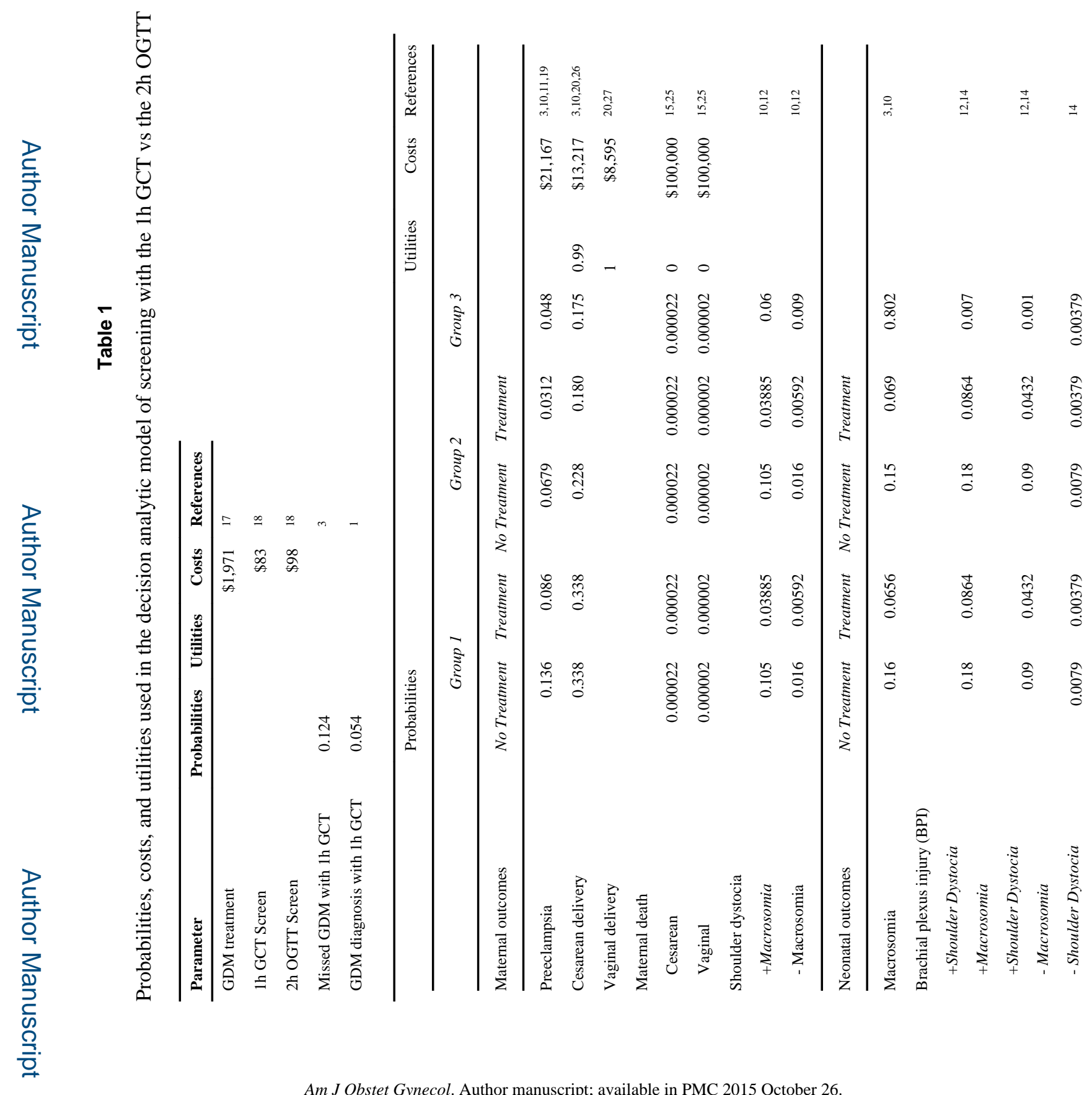




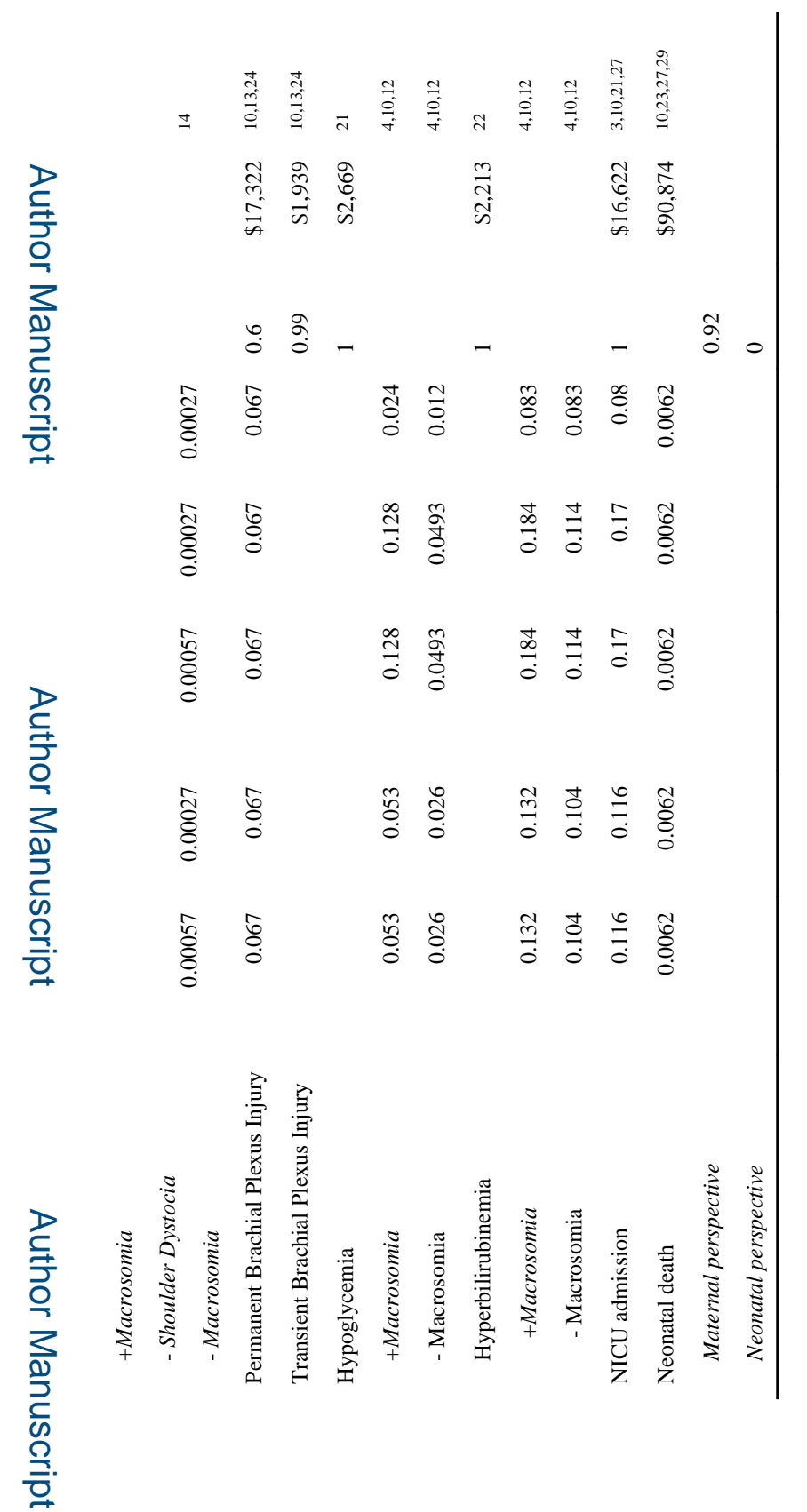




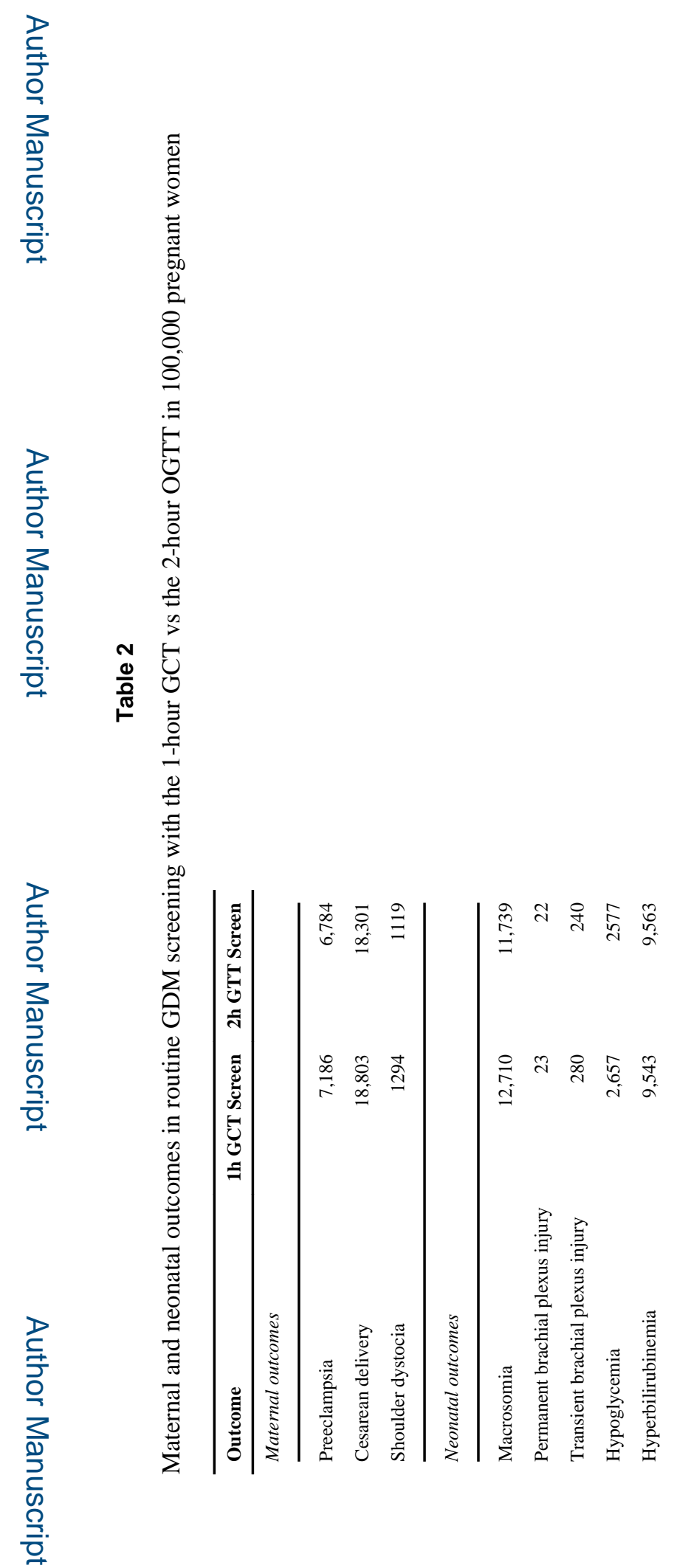




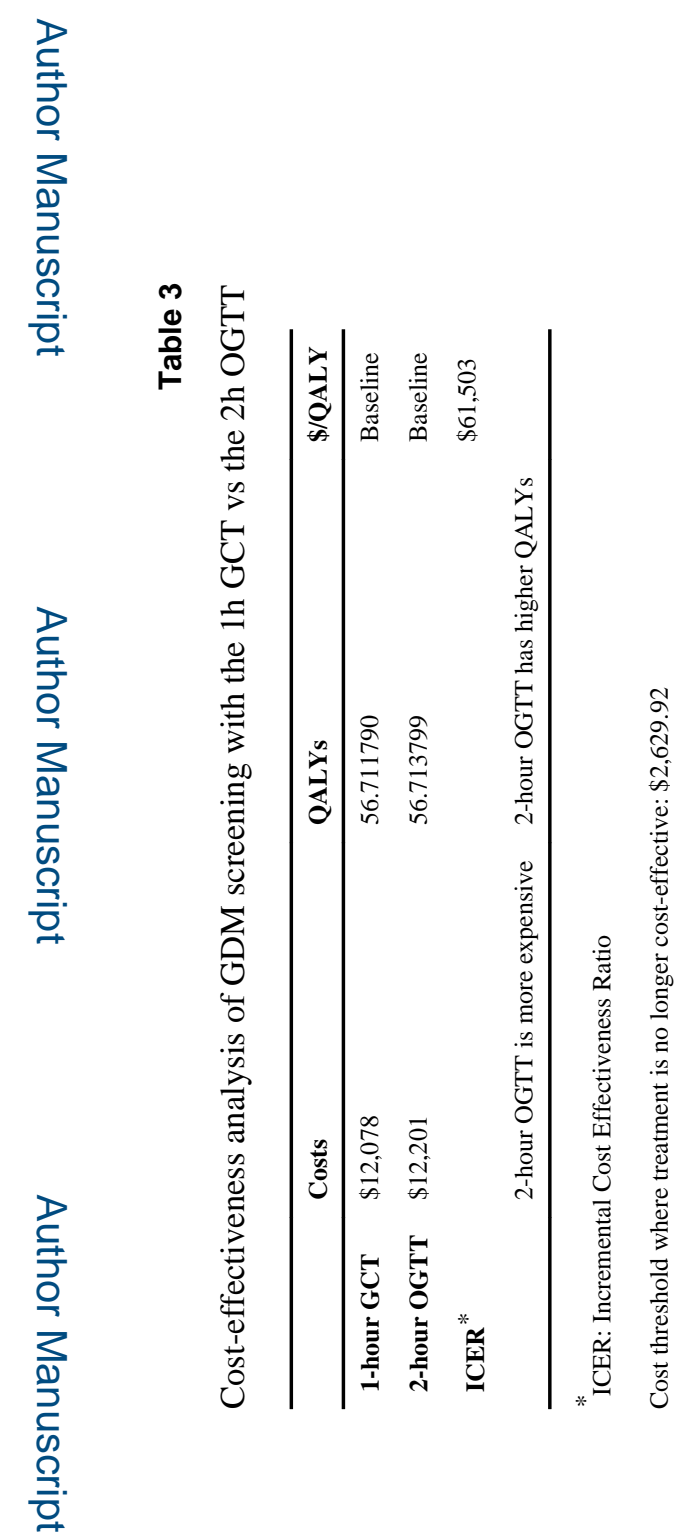

Am J Obstet Gynecol. Author manuscript; available in PMC 2015 October 26. 